\title{
Shared decision making in patients with low risk chest pain: prospective randomized pragmatic trial
}

\author{
Erik P Hess, ${ }^{1,2,3}$ Judd E Hollander, ${ }^{4}$ Jason T Schaffer, ${ }^{5}$ Jeffrey A Kline, ${ }^{5}$ Carlos A Torres, ${ }^{6}$ Deborah B Diercks, ${ }^{7}$ \\ Russell Jones, ${ }^{8}$ Kelly P Owen, ${ }^{8}$ Zachary F Meisel, ${ }^{9}$ Michel Demers, ${ }^{10}$ Annie Leblanc, ${ }^{2,11}$ Nilay D Shah,,11 \\ Jonathan Inselman, ${ }^{3}$ Jeph Herrin, ${ }^{13}$ Ana Castaneda-Guarderas, ,2,14 Victor M Montori2,15
}

For numbered affiliations see end of article.

Correspondence to: E P Hess hess.erik@mayo.edu

Additional material is published online only. To view please visit the journal online.

Cite this as: $B M J$ 2016;355:i6165 http://dx.doi.org/10.1136/bmj.i6165

Accepted: 03 November 2016

\section{ABSTRACT}

OBJECTIVE

To compare the effectiveness of shared decision making with usual care in choice of admission for observation and further cardiac testing or for referral for outpatient evaluation in patients with possible acute coronary syndrome.

DESIGN

Multicenter pragmatic parallel randomized controlled trial.

SETTING

Six emergency departments in the United States.

PARTICIPANTS

898 adults (aged $>17$ years) with a primary complaint of chest pain who were being considered for admission to an observation unit for cardiac testing ( 451 were allocated to the decision aid and 447 to usual care), and 361 emergency clinicians (emergency physicians, nurse practitioners, and physician assistants) caring for patients with chest pain.

INTERVENTIONS

Patients were randomly assigned (1:1) by an electronic, web based system to shared decision making facilitated by a decision aid or to usual care. The primary outcome, selected by patient and caregiver advisers, was patient knowledge of their risk for acute coronary syndrome and options for care; secondary outcomes were involvement in the decision to be admitted, proportion of patients admitted for cardiac testing, and the 30 day rate of major adverse cardiac events.

\section{WHAT IS ALREADY KNOWN ON THIS TOPIC}

Current clinical, electrocardiographic, and laboratory tests miss about $1.5 \%$ of patients with acute coronary syndrome

Given the potential for missed diagnosis, clinicians have a low risk threshold to admit patients for observation and advanced cardiac testing, leading to false positive test results, unnecessary downstream procedures, and increased cost to patients and the healthcare system

Evidence for the effectiveness, safety, and acceptability of a shared decision making approach to communicate risk to patients and engage them in decisions about testing and follow up is limited

\section{WHAT THIS STUDY ADDS}

Use of a decision aid increased patient knowledge and engagement, decreased decisional conflict and the rate of admission to an observation unit for advanced cardiac testing, and was acceptable to patients and clinicians

Translating validated risk estimates to practice and engaging patients in care decisions through shared decision making might tailor testing to disease risk in a way that is acceptable to patients, clinicians, and policy makers

\section{RESULTS}

Compared with the usual care arm, patients in the decision aid arm had greater knowledge of their risk for acute coronary syndrome and options for care (questions correct: decision aid, $4.2 v$ usual care, 3.6; mean difference $0.66,95 \%$ confidence interval 0.46 to 0.86), were more involved in the decision (observing patient involvement scores: decision aid, $18.3 \mathrm{v}$ usual care, $7.9 ; 10.3,9.1$ to 11.5), and less frequently decided with their clinician to be admitted for cardiac testing (decision aid, 37\% v usual care, $52 \%$; absolute difference $15 \%$; $P<0.001)$. There were no major adverse cardiac events due to the intervention.

\section{CONCLUSIONS}

Use of a decision aid in patients at low risk for acute coronary syndrome increased patient knowledge about their risk, increased engagement, and safely decreased the rate of admission to an observation unit for cardiac testing.

TRIAL REGISTRATION

ClinicalTrials.gov NCT01969240.

\section{Introduction}

Chest pain is the second most common reason people visit emergency departments for evaluation, accounting for over eight million visits annually in the United States ${ }^{1}$ and an estimated 360000 attendances in England and Wales. ${ }^{2}$ Over the past decade the proportion of visits to an emergency department for chest pain decreased by $10 \%$ and the proportion of patients with a diagnosis of acute coronary syndrome in the emergency setting decreased from $26 \%$ to $13 \%$. Despite the decreasing incidence of acute coronary syndrome, advanced cardiac imaging for chest pain has increased nearly fourfold. ${ }^{3}$

Current clinical, electrocardiographic, and laboratory data do not identify all patients with acute coronary syndrome who present to the emergency department, resulting in a $1.5 \%$ miss rate. ${ }^{4}$ Given the potential medical, legal, and psychological sequelae associated with missing such a diagnosis, clinicians have a low threshold to admit patients for prolonged observation and advanced cardiac testing. ${ }^{5}$ As a consequence, low risk patients are often admitted for observation and cardiac stress testing or coronary computed tomography angiography (CCTA). This results in unnecessary hospital admissions, ${ }^{6}$ false positive test results, and unnecessary invasive downstream investigations, at an estimated cost to the healthcare system of over $\$ 7 \mathrm{~b}$ (£5.6b; €6.5b) annually. ${ }^{7}$

To assist clinicians, and patients with possible acute coronary syndrome in making risk informed shared 
decisions about testing and follow-up, we included validated $^{89} 45$ day risk estimates for acute coronary syndrome into a decision aid, "chest pain choice."10 In a single center pilot randomized trial of this decision aid, we observed increased patient knowledge about risk of acute coronary syndrome, increased patient engagement, decreased decisional conflict, and a 19\% lower rate of admission to an observation unit for cardiac stress testing in the decision aid compared with the usual care arm, with no adverse events in either study arm. ${ }^{11}$ This pilot randomized trial was conducted in a single tertiary care academic emergency department in the central United States. To test the effectiveness of the decision aid to improve patient knowledge and decrease unnecessary resource use in a broader population of patients with greater socioeconomic diversity and in a variety of clinical contexts, we conducted a multicenter pragmatic $^{12}$ randomized trial in six geographically diverse emergency departments across the United States.

\section{Methods}

\section{Study design}

The background and methods of the trial have been described previously. ${ }^{13}$ This was a pragmatic parallel randomized controlled trial in low risk patients presenting to the emergency department with a potential acute coronary syndrome. The trial compared an intervention group receiving a structured risk assessment using a quantitative pretest probability web tool ${ }^{14}$ and corresponding decision aid with a control group receiving usual care. ${ }^{15}$ Patients and clinicians were enrolled from the emergency departments at six US sites (University of California Davis on the west coast, Mayo Clinic Rochester and Indiana University in central US, University of Pennsylvania and Thomas Jefferson University on the east coast, and Mayo Clinic Florida in the southeast). All of the sites, with the exception of Mayo Clinic Florida, had access to an emergency department observation unit in which protocols to provide care for patients with potential acute coronary syndrome existed as part of routine practice.

\section{Participants}

Eligible clinicians included all emergency physicians, nurse practitioners, and physician assistants caring for patients with chest pain. Eligible patients included adults (aged $>17$ years) presenting to the emergency department with a chief complaint of chest pain who were being considered by the treating clinician for admission to the observation unit for cardiac stress testing or CCTA. Patients were excluded if they had ischemic changes on the initial ECG (eg, ST segment depression, T wave inversion, or new left bundle branch block), had an initial cardiac troponin level more than the 99th centile, had known coronary artery disease, had used cocaine in the past 72 hours (by history or testing), had a prior plan for cardiac intervention or admission, had barriers to outpatient follow-up, were prisoners, were pregnant, were hearing or visually impaired, or were otherwise unable to use the decision aid. We classified patients deemed to meet the exclusion criteria after randomization, but before the patient-clinician disposition discussion as post-randomization exclusions. ${ }^{16}$

\section{Randomization and masking}

Allocation was concealed by an online password protected randomization algorithm (Medidata Balance; Medidata Solutions, New York City, NY). Patients were randomized 1:1 and dynamically stratified ${ }^{17}$ by age, sex, and site because of the known associations of age and sex with cardiovascular risk, potential unmeasured differences between sites, and the availability of these data at the time of enrollment. Clinicians were not randomized. Patients, study coordinators, and treating clinicians were not masked to allocation. All other investigators were blinded to allocation.

\section{Study treatments \\ Intervention}

We sought to assist patients and clinicians in making a risk informed shared decision in the emergency setting in which patients typically do not have the opportunity to learn about their condition prior to the visit and clinicians often make decisions unilaterally to facilitate patient safety and rapid treatment of life threatening conditions. For these contextually specific reasons, we designed the decision aid for use during the clinical encounter. ${ }^{18}$ The decision aid was developed ${ }^{10}$ in Rochester, Minnesota through a participatory action research methodology ${ }^{19}$ in which feedback was intentionally and iteratively sought from patients, clinicians, an expert in healthcare design, and the investigative team and was field tested until thematic saturation was achieved. Prior to conducting the trial, we refined the decision aid to ensure contextual fit with each practice setting. Figure 1 shows the refined decision aid. At two of the sites, CCTA was available and frequently carried out in the evaluation of patients with possible acute coronary syndrome. For these two sites, the option of CCTA was added to the decision aid (see supplementary figure).

\section{Delivery of the intervention}

For patients randomized to the decision aid, a study coordinator collected each of the variables needed to populate the quantitative probability web tool, ${ }^{14}$ asked the treating clinician to sign off on their accuracy, and calculated the patient's pretest probability of acute coronary syndrome, incorporating the result of the first troponin test but prior to subsequent biomarker testing (fig 2). After selecting the decision aid corresponding to the appropriate level of risk, the study coordinator offered to provide the clinician with a concise refresher of the content. The treating clinician, after evaluating the patient and the results of the initial ECG and cardiac troponin tests, then used the decision aid to educate the patient about the results of the two tests, the potential need for observation and further cardiac testing, subsequent cardiac troponin testing to definitively rule out acute myocardial infarction, if required, and their 
1 Your Chest Pain Diagnosis Your initial test results are NEGATIVE
for a heart attack. These included:

- Blood tests to look for an enzyme called troponin that is released when the heart muscle is damaged. Additional troponin tests may be done to monitor you for heart attack during your emergency visit.

- An electrocardiogram to check whether your heart is getting enough oxygen and blood.

However, the chest pain you are experiencing today may be a warning sign for a future heart attack.

2 What You Can Do

2 A STRESS TEST, which views blood flow to your heart at rest and under stress may be needed.

Examining your risk will help you and your clinician decide together whether or not you should have additional heart testing.

'Stress test options include nuclear stress testing Itrasound stress testing, or exercise ECG (electrocardiogram) stress testing. Nuclear stress esting involves exposure to radiation which has been shown to be related to increased cancer risk over a lifetime. Your doctor can help you explore which option may be best for you.

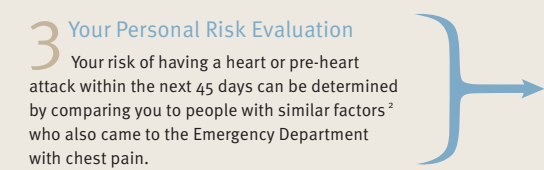

Of every 100

people like yo who came to the Emergency Department with chest pain

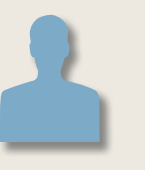

3 had a heart or a pre-heart attack within 45 days of their Emergency Department visit,

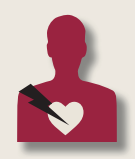
97 did not.

Fig 1 | Decision aid to facilitate discussion between clinicians and patients on whether to be admitted to an observation unit in the emergency department for cardiac stress testing or to follow up with a clinician in 24-72 hours

\section{PRETEST CONSULT}

\section{ACS Pretest Probability \\ Assessment (Version 2.10)}

INDICATIONS: The PreTest Consult instrument is intended for prescription use in a hospital, emergency department or urgent care environment by competent health professionals. The PreTest Consult utilizes clinical variables and ECG data to produce a numerical score that is the pretest probability of acute physician's decision-making process. The advinten fed to supplement, not substitute for the physician's decision-making process for possible or suspected acute cardiac ischemia or pulmonary peris and other clinical findings.

\section{Age}

Gender

Race

Chest Pain

Reproduced by

Palpation?

\section{Personal History of CAD?}

Diaphoresis?

EKG ST Depression > $0.5 \mathrm{~mm}$ ?

T Wave Inversion Deeper than -0.5 ?

\begin{tabular}{|l|}
\hline $150 \quad \hat{v}$ \\
\hline$O M \odot F$ \\
\hline African American $\hat{v}$ \\
\hline$\bigcirc$ Yes $\odot$ No \\
\hline \hline Y Yes $\odot$ No \\
\hline \hline Yes $\odot$ No \\
\hline \hline Yes $\odot$ No \\
\hline \hline$O$ Yes $\odot$ No \\
\hline
\end{tabular}

\begin{tabular}{|lll|}
\hline \multicolumn{3}{|c|}{45 Day Post test Probabilities of ACS } \\
& Neg. & Pos. \\
CKMB $(5 \mathrm{ng} / \mathrm{mL})$ & $\mathbf{2 . 1} \%$ & $\mathbf{2 9 \%}$ \\
Troponin I $(0.4 \mathrm{ng} / \mathrm{mL})$ & $\mathbf{2 \%}$ & $\mathbf{2 1 \%}$ \\
Troponin T $(0.1 \mathrm{ng} / \mathrm{mL})$ & $\mathbf{2 . 3 \%}$ & $\mathbf{1 8 . 3} \%$ \\
myoglobin $(70 \mathrm{ng} / \mathrm{mL})$ & $\mathbf{2 \%}$ & $\mathbf{1 7 . 0} \%$ \\
myoglobin \& Troponin & $\mathbf{0 . 9} \%$ & $\mathbf{1 4 . 6 \%}$ \\
\hline
\end{tabular}

\begin{tabular}{|l|l|}
\hline Get Pretest Prob & Get Post Test Probs \\
\hline Print Results \\
\hline
\end{tabular}

Fig 2 | Screen shot of quantitative pretest probability web tool. Figure displays 45 day probability of acute coronary syndrome for an African-American woman aged more than 50 years whose chest pain is not reproducible with palpation, is not diaphoretic, and there is no ST segment depression greater than $0.5 \mathrm{~mm}$ or T wave inversion deeper than $-0.5 \mathrm{~mm}$, incorporating the result of the first cardiac troponin test. In this case, a coordinator would select a decision aid demonstrating a 3 out of 100 risk, rounding up from $2.3 \%$ to prioritize patient safety 
personalized 45 day risk for acute coronary syndrome. The clinician then engaged the patient in selecting the management option most closely aligned to his or her values and preferences.

\section{Usual care}

For patients randomized to usual care, a study coordinator instructed the clinician to discuss the results of diagnostic investigations and management options according to the clinician's usual manner. Clinicians treating patients in the usual care arm did not have access to the quantitative probability web tool or to the decision aid. As the trial was intentionally pragmatic in design, usual care was not standardized. ${ }^{12}$

\section{Data collection}

We collected data documenting the screening process, randomization, and outcome assessment in compliance with CONSORT guidelines. ${ }^{20}$ Data on patient knowledge of their risk for acute coronary syndrome and the available management options, decisional conflict, and patients' trust in their physician were collected by immediate post-visit survey. ${ }^{13}$ The clinician-patient discussion was video and audio recorded..$^{21}$ Video and audio recordings were time stamped, and we determined the duration of the clinician-patient discussion from these recordings. The recordings were uploaded to a secure server and deleted from the portable devices after upload. Access to the server was protected by a two step access: password protected access to all Mayo's computers, and password protected access to the secure server. Audio and video files from facilities outside of Mayo Clinic were downloaded onto a password protected flash drive, sent to the prime site by secure courier, and uploaded to a secure server on receipt. We collected data on cardiac risk factors, post-emergency department management, and further cardiac investigations by review of the electronic medical record at each site.

Study coordinators contacted patients, starting at 45 days after enrollment, to assess utilization and safety. Study coordinators made at least five attempts to contact patients by phone for follow-up during different times of the day and on different days of the week. If patients were unable to be reached by phone or email and no subsequent visits in the electronic medical record were documented, mortality status was verified using Accurint, a national database frequently used by banks and other businesses to track individuals and ensure payment collection..$^{22}$

\section{Patient involvement}

Patients seeking emergency care for chest pain, a patient adviser (MD), and a caregiver adviser (AL) were involved in the design of the study, the design of the intervention, submission of the application for funding, monitoring of study conduct, interpretation of the data, review of the manuscript for important intellectual content, and approval of the final manuscript for publication. When designing the trial, the patient adviser, caregiver adviser, and emergency department patient advisory council at the Saint Marys Hospital at Mayo Clinic provided input on the prioritization and selection of outcomes. As the primary purpose of the study was to educate and empower patients to participate in decisions about their emergency care, the patient's viewpoint was prioritized over outcomes of potential interest to other stakeholders. Outcomes of interest to other key stakeholders were included as secondary outcomes. When designing the intervention, input was sought from the patient and caregiver advisers, the emergency department patient advisory council, and patients receiving emergency care for potential acute coronary syndrome regarding the clarity, helpfulness, and usefulness of the information included in the decision aid, and the decision aid was iteratively refined based on this input. As the patients and patient and caregiver advisers involved in the trial had no prior diagnosis of coronary artery disease and thus no engagement in a heart disease specific support group or organization, patient advisers were not directly involved in dissemination of the study findings. However, the patient and caregiver advisers were engaged at the highest level possible-partner-and included as co-investigators on the application for funding, members of the investigative steering committee, and assisted in interpretation of the data, review of the final manuscript for important intellectual content, and approval of the final manuscript for submission, thus meeting the criteria for authorship and inclusion in the manuscript as coauthors.

\section{Outcomes \\ Primary outcome}

As the goal of research on patient centered outcomes is to provide patients and the public with the information they need to help them make decisions that affect their desired health outcomes, ${ }^{23}$ we prioritized the perspective of the patient over the perspectives of the other stakeholders in determining the primary outcome. During meetings with patient and caregiver advisers, knowledge about risk of acute coronary syndrome emerged as the outcome of greatest importance, so we selected patient knowledge as the primary outcome. As done in our pilot trial ${ }^{11}$ and in prior work, ${ }^{24}$ we assessed patient knowledge by immediate post-visit survey (see supplementary file).

\section{Secondary outcomes}

We measured the degree of uncertainty patients experienced related to feeling uninformed about the management options using the decisional conflict scale ${ }^{25}$ and patient trust in their clinician using the trust in physician scale. ${ }^{26}$ The decisional conflict scale includes 16 items that are scored from 0-4; the items are summed, divided by 16 , and then multiplied by 25 . The scale is from 0-100, where higher scores are reflective of increased patient uncertainty about the choice. One study found that for every unit increase in decisional conflict scale scores, patients were 19\% more likely to blame their doctor for bad outcomes. ${ }^{27}$ As such, a 1 unit change in decisional conflict scale score is considered 
clinically meaningful. The trust in physician scale consists of nine items scored from 1-5; the items are subtracted by 1 , summed, divided by 9 , and then multiplied by 25 . The scale ranges from $0-100$, where higher values are reflective of higher levels of patient trust in their physician. To the best of the authors' knowledge, a clinically meaningful change in trust in physician scale score has not been published. We surveyed participating patients and clinicians about the clarity and helpfulness of the information shared and the acceptability of the decision aid using a 7 point Likert scale. Finally, five trained raters independently viewed videos of the patient-clinician discussion and assessed the degree to which clinicians engaged patients in the decision making process using the observing patient involvement (OPTION) scale. ${ }^{21}$ This scale is composed of 12 items with a value of $0-4$; they are summed, divided by 48 , and then multiplied by 100 . Scores range from $0-100$, where higher scores are reflective of higher levels of patient engagement. Although a clinically meaningful change in OPTION scale score has not been defined, the mean score for outpatient clinicians in the original development investigation was 16.9 (SD 7.68). ${ }^{28}$ Given that the current trial was conducted in the emergency setting, in which time pressures and patient acuity often impact the clinician-patient interaction, we anticipated OPTION scale scores in the current investigation to be lower than the originally published mean.

We assessed the acceptability of the decision aid by immediate post-visit survey. ${ }^{13}$ Patients in both the decision aid and usual care arms were asked to rate the amount, clarity, and helpfulness of the information they received and whether they would want to get information in the same way and would recommend the way that they and their provider shared information about their chest pain symptoms and options for care. Responses were recorded using a 7 point Likert scale.

We assessed management by recording whether patients were admitted to the observation unit of the emergency department, admitted to hospital, or discharged home; whether cardiac stress testing or CCTA were done; the results of testing; and whether the patient underwent percutaneous coronary intervention or coronary artery bypass grafting. The data were collected by review of the electronic medical record at each participating site.

To assess safety we determined whether a patient experienced a major adverse cardiac event (MACE). Consistent with a consensus document on acute coronary syndrome research in emergency departments, ${ }^{29}$ we defined MACE as acute myocardial infarction, ${ }^{30}$ death due to a cardiac or unknown cause, emergency revascularization, ventricular arrhythmia, or cardiogenic shock. Potential MACEs were shared with the data safety monitoring board and discussed by the entire investigative team during monthly conference calls, and adjudicated based on consensus among site investigators. We excluded MACE occurring during the index visit to the emergency department or hospital, as these events were considered appropriately diagnosed during that visit. Events occurring after discharge home, which could have potentially been avoided, were classified as MACE. We collected data on all MACE occurring up to 45 days to be consistent with the follow-up period used in the development of the quantitative pretest probability instrument, ${ }^{31}$ but we compared 30 day event rates to comply with standardized reporting guidelines for emergency department risk stratification studies of patients with potential acute coronary syndrome. ${ }^{32}$ We plan to report the effect of the decision aid on healthcare utilization in a separate manuscript.

\section{Clarification of primary outcome}

The primary outcome registered at clinicaltrials.gov is the phrase "Test if Chest Pain Choice [the decision aid] safely improves validated patient-centered outcome measures" with the description "Test if the intervention significantly increases patient knowledge." There is only one primary outcome for the study: patient knowledge. The phrase "Test if Chest Pain Choice safely improves validated patient-centered outcome measures" refers to the five additional outcome measures listed as secondary outcomes at clinicaltrials.gov (a through e) and is redundant. This is documented in the study protocol, ${ }^{13}$ which was published prior to completion of enrollment for the trial in August 2015.

\section{Statistical analysis}

We estimated that 884 patients would provide 99\% power to detect a $16 \%$ difference in patient knowledge between decision aid and usual care arms and 90\% power to detect a $10 \%$ difference in the proportion of patients admitted to an observation unit for cardiac testing. ${ }^{13}$ To account for an estimated 5\% potential loss to follow-up, we planned to enroll 930 patients. We summarized patient characteristics by study group and tested for differences between groups using $t$ tests and $\chi^{2}$ tests. To test for differences in outcomes, we estimated a series of regression models, each of which included indicators for study group. For continuous outcomes we used linear models, and for categorical outcomes we used multinomial (polytomous) logistic models. To account for non-independence of outcomes by site, we included indicators for study site in each model. We assessed for additional correlation within clinicians by estimating a hierarchical generalized model for each outcome and calculating the intra-clinician correlation. All intra-clinician correlations were less than $1 \%$, so we chose not to account for this correlation in the final models. We followed the principle of intention to treat in the conduct of the trial and in all analyses.

\section{Results}

We assessed 3236 patients for eligibility from October 2013 to August 2015 (fig 3). Overall, 361 clinicians participated in the study. In total, 913 patients were randomized, with 13 post-randomization exclusions and two patients who withdrew consent, leaving 898 patients (451 in the decision aid arm, 447 in the usual care arm) in the final analysis. In all 13 post-randomization 


\section{Assessed for eligibility $(n=3236)$}

\begin{tabular}{|c|c|c|}
\hline & \multicolumn{2}{|c|}{$\begin{array}{l}\text { Excluded }(n=2323) \text { : } \\
\text { Not meeting inclusion criteria }(n=2012) \text { : } \\
\text { Known coronary artery disease }(n=1034) \\
\text { Acute ischemia on initial electrocardiogram (ECG) }(n=340) \\
\text { Unable to use decision aid (for example, learning barrier, dementia) ( } n=302) \\
\text { Troponin }>99 \text { th centile }(n=137) \\
\text { Cocaine use within } 72 \text { hours }(n=65) \\
\text { Prior plan for cardiac intervention or admission }(n=62) \\
\text { Barriers to outpatient follow-up }(n=57) \\
\text { Prisoner }(n=13) \\
\text { Pregnant }(n=2) \\
\text { Patient refused to participate }(n=166) \\
\text { Provider refused to participate }(n=130) \\
\text { Other reasons }(n=15)\end{array}$} \\
\hline \multicolumn{3}{|c|}{ Randomized $(n=913)$} \\
\hline & 1 \\
\hline \multicolumn{2}{|c|}{$\begin{array}{l}\text { Allocated to intervention }(n=458) \text { : } \\
\text { Received allocated intervention }(n=451) \\
\text { Post-randomization exclusions }(n=6) \text { : } \\
\text { New ischemia on ECG }(n=2) \\
\text { Stress testing no longer being considered }(n=2) \\
\text { Initial troponin }>99 \text { th centile }(n=1) \\
\text { Positive test for cocaine }(n=1) \\
\text { Patient withdrew consent }(n=1)\end{array}$} & $\begin{array}{l}\text { Allocated to usual care }(n=455) \text { : } \\
\text { Received allocated intervention }(n=447) \\
\text { Post-randomization exclusions }(n=7) \text { : } \\
\text { New ischemia on ECG }(n=2) \\
\text { Stress testing no longer being considered }(n=1) \\
\text { Initial troponin }>99 \text { th centile }(n=1) \\
\text { Known coronary artery disease }(n=1) \\
\text { Patient withdrew consent }(n=1)\end{array}$ \\
\hline & $\downarrow$ & $\downarrow$ \\
\hline \multicolumn{2}{|c|}{$\begin{array}{l}\text { Contacted by phone or email at } 45 \text { days }(n=413) \\
\text { Mortality assessed by electronic medical } \\
\text { record or Accurint database }(n=38)\end{array}$} & $\begin{array}{l}\text { Contacted by phone or email at } 45 \text { days }(n=415) \\
\text { Mortality assessed by electronic medical } \\
\text { record or Accurint database }(n=30) \\
\text { Mortality status unable to be verified }(n=2)\end{array}$ \\
\hline \multicolumn{2}{|r|}{$\nabla$} & $\downarrow$ \\
\hline \multicolumn{2}{|c|}{$\begin{array}{l}\text { Included in final analysis }(n=451) \\
\text { Post visit patient surveys completed }(n=444) \\
\text { Post visit clinician surveys completed }(n=436) \\
\text { Recorded encounters included in videographic } \\
\text { analysis }(n=264)\end{array}$} & $\begin{array}{l}\text { Included in final analysis }(n=447) \\
\text { Post visit patient surveys completed }(n=442) \\
\text { Post visit clinician surveys completed }(n=430) \\
\text { Recorded encounters included in videographic } \\
\text { analysis }(n=272)\end{array}$ \\
\hline
\end{tabular}

Fig 3 | Participant flow diagram
$4.2 v$ usual care, 3.6; mean difference $0.66,95 \%$ confidence interval 0.46 to 0.86 ; table 2 ). A greater proportion of patients in the decision aid arm correctly reported their exact pretest probability of acute coronary syndrome and their risk within $10 \%$ of the correct value (decision aid, $65.0 \% \mathrm{v}$ usual care, $18.1 \%$; absolute difference $46.8 \%$, 95\% confidence interval $41.2 \%$ to $52.5 \%$ ). Patients in the decision aid arm reported significantly less decisional conflict (decision conflict scale: decision aid, 43.5 (SD 15.3) v usual care, 46.4 (SD 14.8); mean difference $-2.9,-4.8$ to -0.90$)$. Use of the decision aid did not significantly impact patients' trust in their physician. The proportion of patients who were "strongly satisfied" with the decision aid was not significantly different between study arms (decision aid, $49 \% v$ usual care, 43\%; absolute difference 6\%, $\mathrm{P}=0.06)$.

\section{Patient participation and acceptability}

Interobserver agreement between raters for OPTION scale assessments was 0.89 (95\% confidence interval 0.84 to 0.93 ). Patients randomized to the decision aid were more engaged in the decision making process, as indicated by higher OPTION scores (decision aid, 18.3 (SD 9.4) $v$ usual care, 7.9 (5.4); mean difference 10.3, 9.1 to 11.5; table 2). Patients randomized to the decision aid found the information discussed to be of greater clarity, and a greater proportion (decision aid, 88.0\% $v$ usual care, $79.9 \%$; absolute difference $8.1 \%, \mathrm{P}=0.004$ ) would recommend the way they discussed management options with their clinician to others.

\section{Clinician acceptability}

A greater proportion of clinicians in the decision aid arm found the information to be extremely helpful (table 2). Most ( $\mathrm{n}=217,62.7 \%$ ) clinicians would recommend the decision aid to others, and 62.9\% $(n=273)$ would want to use a decision aid for other decisions. The mean length of the discussion was 1.3 minutes longer in the decision aid arm (decision aid, 4.4 (SD 0.40 ) minutes $v$ usual care, 3.1 (0.29) minutes; mean difference $1.3, \mathrm{P}=0.008$ ).

\section{Management and 30 day outcomes}

A significantly lower proportion of patients randomized to the decision aid decided, with their clinician, to be admitted to the emergency department observation unit for cardiac stress testing or CCTA (decision aid, 37.3\% v usual care, 52.1\%; absolute difference 14.8\%, $95 \%$ confidence interval $1.1 \%$ to $13.9 \%$ ), and a significantly lower proportion underwent cardiac stress testing within 30 days (decision aid, 38.1\% v usual care, $45.6 \%$; $7.5 \%, 1.1 \%$ to $13.9 \%$; table 3 ). There was no significant difference between arms in the proportion of patients who opted to follow up with a cardiologist or primary care provider and did not have a stress test or outpatient visit within 30 days (2/249 (0.8\%) decision aid $v$ 4/152 (2.6\%) usual care; Fisher's exact test $\mathrm{P}=0.20$ ). Of those who underwent cardiac stress testing, a significantly greater proportion of patients randomized to the decision aid had testing performed in the outpatient 


\begin{tabular}{|c|c|c|}
\hline Characteristic & Usual care $(n=447)$ & Decision aid $(n=451)$ \\
\hline Mean (SD) age (years) & $50.6(14.1)$ & $50.0(15.0)$ \\
\hline Median (interquartile range) age (years) & $51.0(44.0-59.0)$ & $51.0(43.0-58.0)$ \\
\hline Women & $260(58.2)$ & $274(60.8)$ \\
\hline \multicolumn{3}{|l|}{ Race: } \\
\hline American Indian/Alaska Native & $4(0.9)$ & $4(0.9)$ \\
\hline Asian & $9(2.0)$ & $6(1.3)$ \\
\hline Black or African-American & $154(34.5)$ & $155(34.4)$ \\
\hline Native Hawaiian or other Pacific Islander & $0(0.0)$ & $2(0.4)$ \\
\hline White & $269(60.2)$ & $262(58.1)$ \\
\hline Other & $11(2.5)$ & $22(4.9)$ \\
\hline \multicolumn{3}{|l|}{ Annual income $(\$)(n=850)$ : } \\
\hline$<20000$ & $84(18.8)$ & $92(20.4)$ \\
\hline $20000-30000$ & $39(8.7)$ & $36(8.0)$ \\
\hline $30000-40000$ & $46(10.3)$ & $44(9.8)$ \\
\hline $40000-60000$ & $65(14.5)$ & $56(12.4)$ \\
\hline $60000-80000$ & $59(13.2)$ & $58(12.9)$ \\
\hline $80000-100000$ & $37(8.3)$ & $50(11.1)$ \\
\hline$>100000$ & $93(20.8)$ & $100(22.2)$ \\
\hline \multicolumn{3}{|l|}{ Highest level of education completed $(n=881)$ : } \\
\hline High school or less & $38(8.5)$ & $47(10.4)$ \\
\hline High school or graduate education diploma & $109(24.4)$ & $91(20.2)$ \\
\hline College or vocational school & $157(35.1)$ & $150(33.3)$ \\
\hline College graduate ( 4 years) & $82(18.3)$ & $98(21.7)$ \\
\hline Graduate degree & $54(12.1)$ & $55(12.2)$ \\
\hline \multicolumn{3}{|l|}{ Literacy screening questions (often/always): } \\
\hline Need help reading medical instructions? & $36(8.1)$ & $44(9.8)$ \\
\hline Confident filling out medical forms? & $358(80.1)$ & $375(83.1)$ \\
\hline Have difficulty understanding written information about your medical condition? & $28(6.3)$ & $40(8.9)$ \\
\hline Mean (SD) subjective numeracy scale score* & $4.3(1.1)$ & $4.2(1.1)$ \\
\hline Median (interquartile range) numeracy & $4.4(3.5-5.1)$ & $4.4(3.4-5.1)$ \\
\hline Hypertension & $202(45.1)$ & $198(43.9)$ \\
\hline Dyslipidemia & $137(30.6)$ & $114(25.3)$ \\
\hline Diabetes mellitus & $71(15.8)$ & $61(13.5)$ \\
\hline Family history of cardiac disease & $182(40.6)$ & $176(39.0)$ \\
\hline Smoking (current, recent cessation, or former) & $165(36.8)$ & $181(40.1)$ \\
\hline Renal insufficiency & $9(2.0)$ & $7(1.6)$ \\
\hline History of stroke or transient ischemic attack & $16(3.6)$ & $18(4.0)$ \\
\hline Mean (SD) duration of chest pain (hours) & $3.3(5.5)$ & $3.1(5.0)$ \\
\hline Median (interquartile range) duration of chest pain (hours) & $1.0(0.3-4.0)$ & $1.0(0.2-3.5)$ \\
\hline Mean (SD) probability of ACSt & $3.8(4.3)$ & $3.6(3.7)$ \\
\hline Median (interquartile range) probability of ACS & $2.8(0.6-5.2)$ & $2.8(0.6-5.2)$ \\
\hline Friend or family member present & $244(54.6)$ & $257(57.0)$ \\
\hline $\begin{array}{l}\$ 1.0 \text { (£0.8; €0.9). } \\
\text { ACS=acute coronary syndrome. } \\
\text { *McNaughton et al. }{ }^{41} \\
\text { +Calculated from quantitative probability web tool. }\end{array}$ & & \\
\hline
\end{tabular}

setting (decision aid, 30.2\% $v$ usual care, 17.2\%; 13.1\%, $4.5 \%$ to $21.7 \%$ ). The rate of coronary angiography, coronary revascularization, admission to hospital, readmission to hospital, repeat emergency department visits, or outpatient clinic visits did not differ between study arms.

Four of the five patients with acute myocardial infarction and all cardiac interventions occurred during the index visit. Three of the four patients with acute myocardial infarction in the decision aid arm had an initial troponin level less than the 99th centile, no acute ischemic changes on the initial ECG, and a subsequent increased troponin level detected on serial cardiac biomarker testing. These patients were admitted to the hospital for further evaluation and management and received a diagnosis of non-ST segment elevation myocardial infarction. The fourth case of myocardial infarction in the decision aid arm occurred in a patient who had negative serial cardiac troponin results and no acute ischemic changes on the ECG but symptoms suggestive of acute coronary syndrome. This patient was admitted to the hospital, underwent percutaneous coronary intervention, and subsequently developed in-stent thrombosis. This in-stent thrombosis, which occurred in the hospital, was accompanied by increased troponin levels and ST segment elevation on ECG. The patient underwent a second percutaneous coronary intervention and recovered uneventfully. 


\begin{tabular}{|c|c|c|c|}
\hline Outcome & $\begin{array}{l}\text { Usual care } \\
(n=447)\end{array}$ & $\begin{array}{l}\text { Decision aid } \\
(n=451)\end{array}$ & $\begin{array}{l}\text { Mean difference } \\
(95 \% \mathrm{Cl}) \text { or } \mathrm{P} \text { value }\end{array}$ \\
\hline \multicolumn{4}{|l|}{ Patient knowledge } \\
\hline Eight knowledge questions & $3.6(1.5)$ & $4.2(1.5)$ & 0.66 (0.46 to 0.86$)$ \\
\hline Correctly assessed 45 day risk for ACS & $2(0.4)$ & $10(2.2)$ & 0.039 \\
\hline Correctly assessed 45 day risk for ACS within $10 \%$ & $81(18.1)$ & $293(65.0)$ & $<0.001$ \\
\hline \multicolumn{4}{|l|}{ Decisional conflict and trust } \\
\hline Decisional conflict scale & $46.4(14.8)$ & $43.5(15.3)$ & $-2.9(-4.8$ to -0.90$)$ \\
\hline Trust in physician scale & $87.7(16.0)$ & $89.5(13.4)$ & $1.7(-0.2$ to 3.6$)$ \\
\hline \multicolumn{4}{|l|}{ Patient involvement in decision } \\
\hline OPTION scale $(n=536)$ & $7.9(5.4)$ & $18.3(9.4)$ & $10.3(9.1$ to 11.5$)$ \\
\hline \multicolumn{4}{|l|}{ Patient acceptability } \\
\hline \multicolumn{4}{|l|}{ Amount of information: } \\
\hline Too little (1-2) & $24(5.5)$ & $12(2.7)$ & \multirow{3}{*}{0.133} \\
\hline Just right (3-5) & $401(91.6)$ & $416(94.3)$ & \\
\hline Too much (6-7) & $13(3.0)$ & $13(2.9)$ & \\
\hline \multicolumn{4}{|l|}{ Clarity of information: } \\
\hline Not clear at all (1-2) & $5(1.1)$ & $7(1.6)$ & \multirow{3}{*}{0.011} \\
\hline Somewhat clear (3-5) & $137(31.3)$ & $98(22.3)$ & \\
\hline Extremely clear (6-7) & $296(67.6)$ & $335(76.1)$ & \\
\hline \multicolumn{4}{|l|}{ Helpfulness of the information: } \\
\hline Not helpful at all (1-2) & $10(2.3)$ & $7(1.6)$ & \multirow{3}{*}{0.506} \\
\hline Somewhat helpful (3-5) & $125(28.5)$ & $114(25.9)$ & \\
\hline Extremely helpful (6-7) & $303(69.2)$ & $320(72.6)$ & \\
\hline \multicolumn{4}{|l|}{ Would recommend to others: } \\
\hline Yes $(1-2)$ & $349(79.9)$ & $387(88.0)$ & \multirow{3}{*}{0.004} \\
\hline Not sure (3-5) & $77(17.6)$ & $44(10.0)$ & \\
\hline No $(6-7)$ & $11(2.5)$ & $9(2.0)$ & \\
\hline \multicolumn{4}{|l|}{ Would want to use for other decisions: } \\
\hline Yes $(1-2)$ & $335(76.7)$ & $346(78.6)$ & \multirow{3}{*}{0.813} \\
\hline Not sure $(3-5)$ & $83(19.0)$ & $77(17.5)$ & \\
\hline No $(6-7)$ & $19(4.3)$ & $17(3.9)$ & \\
\hline \multicolumn{4}{|l|}{ Clinician acceptability } \\
\hline \multicolumn{4}{|l|}{ Helpfulness of the information: } \\
\hline Not helpful at all (1-2) & $13(3.1)$ & $24(5.5)$ & \multirow{3}{*}{$<0.001$} \\
\hline Somewhat helpful (3-5) & $265(63.2)$ & $175(40.3)$ & \\
\hline Extremely helpful (6-7) & $141(33.7)$ & $235(54.1)$ & \\
\hline \multicolumn{4}{|l|}{ Would recommend to others: } \\
\hline Yes $(1-2)$ & $175(41.9)$ & $271(62.7)$ & \multirow{3}{*}{$<0.001$} \\
\hline Not sure (3-5) & $234(56.0)$ & $148(34.3)$ & \\
\hline No $(6-7)$ & $9(2.2)$ & $13(3.0)$ & \\
\hline \multicolumn{4}{|l|}{ Would want to use for other decisions: } \\
\hline Yes (1-2) & $183(43.8)$ & $273(62.9)$ & \multirow{3}{*}{$<0.001$} \\
\hline Not sure (3-5) & $229(54.8)$ & $148(34.1)$ & \\
\hline No $(6-7)$ & $6(1.4)$ & $13(3.0)$ & \\
\hline
\end{tabular}

No deaths of cardiac or unknown cause occurred in either arm. One patient in the decision aid arm was classified as having a MACE. This patient decided with their clinician to be admitted to the hospital and underwent nuclear perfusion stress testing as an inpatient. The test result was interpreted as negative. The patient was discharged from hospital but subsequently developed recurrent chest pain and returned to the emergency department within 30 days of hospital discharge with a non-ST segment myocardial infarction. The data safety monitoring board classified this MACE as unrelated to the intervention.

\section{Discussion}

In patients with chest pain who were otherwise being considered for admission to an observation unit and advanced cardiac testing, shared decision making facilitated by a decision aid increased patient knowledge and patient engagement, decreased decisional conflict, and did not significantly affect trust in physicians. The decision aid was found to be acceptable to both patients and physicians, and its use, which took an average of one additional minute of clinician time, decreased the rate of admission to an observation unit for advanced cardiac testing and cardiac stress testing within 30 days of the emergency department visit. No major adverse cardiovascular events (MACEs) were related to the intervention.

\section{Meaning of the study}

Findings from this trial suggest that patients can be effectively educated and engaged in the emergency care setting in decisions about testing and follow-up and that it is feasible to do so in the flow of clinical care. In addition, when risk estimates from validated prediction models are shared with patients, and patients are invited to apply their informed values and preferences to decisions, rates of admission and testing did not increase. Rather, patient centered interventions such as those tested in this trial indicate that patients, when educated and informed of their risk, might choose with their clinician to undergo less extensive evaluation more closely tailored to their personalized risk. Although we observed less extensive evaluation in this trial, use of shared decision making in other scenarios in which lower utilization occurs than that observed in the US might not have similar results. However, health policy and clinical protocols that encourage transparent communication of risk and patient engagement in care decisions have potential to right-size testing to disease risk in a way that is acceptable to patients, clinicians, and policy makers. The findings from this trial are also timely, given health policy ${ }^{33}$ calling for comparative effectiveness research to assist patients, clinicians, purchasers, and policy makers to make more informed healthcare decisions, and the increased focus on patient centered outcomes research at a national and international level. ${ }^{34}$ To our knowledge, this is the first multicenter trial testing the effect of a shared decision making intervention in the emergency setting.

\section{Limitations and strengths of this study}

Several limitations of this trial should be taken into consideration. The quantitative pretest probability web tool $^{89}$ only applies to patients with chest pain. As such, the decision aid cannot be used in patients with potential acute coronary syndrome who present with non-chest pain syndromes (eg, shortness of breath, diaphoresis). In addition, more accurate methods to estimate patient risk, such as those incorporating high sensitivity troponin C assays, are likely to become available. In the future it might be preferable to generate risk estimates with these methods and select the decision aid that corresponds to this level of risk. We used two versions of the decision aid in the trial-one that included the option of coronary computed tomography angiography (CCTA) and one that included only cardiac stress testing. Although this introduced a degree of heterogeneity in the 


\begin{tabular}{|c|c|c|c|}
\hline Characteristic & $\begin{array}{l}\text { Usual care } \\
(n=447)\end{array}$ & $\begin{array}{l}\text { Decision aid } \\
(\mathrm{n}=451)\end{array}$ & $P$ value \\
\hline \multicolumn{4}{|l|}{ Shared management decision: } \\
\hline Observation unit admission for stress testing or CCTA & $225(52.1)$ & $165(37.3)$ & \multirow{4}{*}{$<0.001$} \\
\hline Follow up with a cardiologist & $52(12.0)$ & $101(22.9)$ & \\
\hline Follow up with a primary care physician & $100(23.1)$ & $138(31.2)$ & \\
\hline Have emergency physician decide & $55(12.7)$ & $38(8.6)$ & \\
\hline Cardiac stress test performed within 30 days & $204(45.6)$ & $172(38.1)$ & 0.013 \\
\hline Outpatient stress testing: & $35(17.2)$ & $52(30.2)$ & 0.001 \\
\hline Exercise treadmill testing & 65 (31.9) & $44(25.6)$ & 0.779 \\
\hline Stress echocardiography & $86(42.2)$ & $81(47.1)$ & \\
\hline Nuclear perfusion testing & $39(19.1)$ & $37(21.5)$ & \\
\hline Other & $14(6.9)$ & $10(5.8)$ & \\
\hline CCTA performed within 30 days & $80(17.9)$ & $63(14.0)$ & 0.111 \\
\hline Coronary revascularization & $4(0.9)$ & $7(1.6)$ & 0.366 \\
\hline Percutaneous coronary intervention & $3(75.0)$ & $6(85.7)$ & \\
\hline Coronary artery bypass grafting & $1(25.0)$ & $1(14.3)$ & \\
\hline Admitted to hospital from ED observation unit & $22(4.9)$ & $22(4.9)$ & 0.990 \\
\hline Repeat ED visit & $39(9.3)$ & $52(12.5)$ & 0.156 \\
\hline Readmission to hospital & $19(4.5)$ & $20(4.8)$ & 0.884 \\
\hline Outpatient clinic visit & $259(62.0)$ & $266(64.1)$ & 0.568 \\
\hline \multicolumn{4}{|l|}{ Cardiac events: } \\
\hline Acute myocardial infarction & $1(0.2)$ & $4(0.9)$ & 0.215 \\
\hline Death of cardiac or unknown cause & $0(0.0)$ & $0(0.0)$ & 1.00 \\
\hline MACE within 30 days* & $0(0.0)$ & $1(0.2)$ & 0.998 \\
\hline
\end{tabular}

$\mathrm{CCTA}=$ coronary computed tomography angiography; $\mathrm{ED}=$ emergency department; $\mathrm{MACE}=$. major adverse cardiac event. *Excluding the index event. pilot trial, greater power and precision would be optimal. It is critical that evidence based local practice protocols to definitively rule out acute myocardial infarction with serial cardiac troponin tests be reliably followed in all patients, regardless of the decision to undergo further cardiac stress testing during the index emergency department visit or to follow-up as an outpatient. This is highlighted by the fact that four patients in the decision aid group and one patient in the usual care group were diagnosed as having acute myocardial infarction during their index presentation. Although no patients in this trial were ruled out for acute myocardial infarction using serial cardiac troponin results, were discharged home from the hospital without further testing, or subsequently experienced an acute myocardial infarction before follow-up as an outpatient, this scenario is possible, and a large scale implementation trial is needed to definitively assess safety.

\section{Implications for practice and policy}

The decision aid frames the decision for the patient, provides standardized terminology, and transparently communicates patient risk and the available management options in a manner that many clinicians might find difficult to reproduce without use of the decision aid. Patient centered decision support interventions such as these are designed to facilitate higher quality conversations with patients than typically occur in contemporary emergency care. Moreover, the results of this trial invite clinicians to consider whether our current perception of the degree to which patients are engaged in decision making as part of usual practice respects patient autonomy and supports interaction with professional judgment.

We recommend clinicians consider using the decision aid in patients who present with acute chest pain, no known history of coronary artery disease, and initial negative electrocardiogram and troponin test results, and for whom the clinician is considering further cardiac investigations such as cardiac stress testing or CCTA. The clinician can obtain an estimate of the patient's 45 day pretest probability for acute coronary syndrome and download the decision aid corresponding to the appropriate level of risk at http://shareddecisions.mayoclinic.org/decision-aid-information/ chest-pain-choice-decision-aid/.Write the patient's name in the top left corner, and give the decision aid to the patient for subsequent review. The discussion on shared decision making should also be documented in the medical record. Depending on the local practice setting, the decision aid can be used by the clinician with a specific patient who meets these criteria or might be implemented in the context of a comprehensive risk stratification protocol for patients in the emergency department with potential acute coronary syndrome.

As support for, and interest in, shared decision making in the context of emergency care delivery has increased, ${ }^{35} 36$ questions have arisen about how this might affect liability risk. ${ }^{37}$ Unfortunately, the relation between shared decision making and liability risk cannot be assessed as it is clouded by variation in the 
meaning and implementation of shared decision making. ${ }^{38}$ While use of shared decision making might decrease clinician's liability risk by improving the patient-clinician relationship, enhancing communication (which is often at the root of lawsuits brought against clinicians after an adverse outcome ${ }^{39}$ ), and decreasing the frequency of invasive procedures, ${ }^{40}$ shared decision making might increase liability risk if the care agreed on by the patient and clinician is sensible but perhaps at odds with what other clinicians would have selected without patient input, as the latter is often used to determine "standard of care."

\section{Unanswered questions and future research}

To date, no shared decision making interventions have been made routine and incorporated into clinical protocols and emergency care delivery. While the findings from this multicenter trial suggest that the decision aid might be effective across a variety of clinical settings, further implementation studies are needed to determine how best to incorporate it in care pathways, how emergency clinicians, cardiologists, and primary care clinicians can best work together to ensure incorporation and implementation of informed patient preferences into admission, testing, and follow-up decisions, and how to ensure patient preferences guide decision making both during and after the emergency department encounter. In addition, as time for clinician-patient interaction in the emergency setting is limited and levels of healthcare literacy between patients vary, time efficient approaches to patient activation that involve education and preparation for engagement in shared decisions with clinicians, such as a brief standardized video, should be explored. Interventions designed to ensure communication of the rationale for care decisions to family members who were not present during the emergency department encounter are also needed to ensure effective implementation of the care decisions made. Healthcare policy to encourage, and perhaps incentivize, risk communication and that incorporates informed patient preferences in emergency care decisions about testing and follow-up might also be needed to align financial incentives with the best interests of patients. Finally, a large scale implementation trial might be needed to more definitively test the safety of the intervention.

\section{Conclusion}

Use of a decision aid in patients with low risk chest pain who were otherwise being considered for admission to an observation unit for cardiac stress testing or CCTA increased patient knowledge and patient engagement and decreased decisional conflict. Shared decision making facilitated by the decision aid was perceived to be acceptable to both patients and physicians, and its use decreased the proportion of patients admitted to the observation unit for cardiac testing, with no adverse events related to the intervention.

\section{AUTHOR AFFILIATIONS}

${ }^{1}$ Department of Emergency Medicine, Mayo Clinic College of Medicine, 200 First Street SW, Rochester, MN 55906, USA

${ }^{2}$ Knowledge and Evaluation Research Unit, Mayo Clinic, Rochester, MN, USA
${ }^{3}$ Mayo Clinic Robert D and Patricia E Kern Center for the Science of Healthcare Delivery, Rochester, MN, USA

${ }^{4}$ Department of Emergency Medicine, Thomas Jefferson University, Philadelphia, PA, USA

${ }^{5}$ Department of Emergency Medicine, Indiana University, Indianapolis, IN, USA

${ }^{6}$ Department of Emergency Medicine, Mayo Clinic, Jacksonville, FL, USA

${ }^{7}$ Department of Emergency Medicine, University of Texas Southwestern Medical Center, Dallas, TX, USA

${ }^{8}$ Department of Emergency Medicine, University of California Davis Medical Center, Sacramento, CA, USA

${ }^{9}$ Department of Emergency Medicine, Perelman School of Medicine, Philadelphia, PA, USA

${ }^{10}$ Rochester, MN, USA

${ }^{11}$ Division of Health Care Policy and Research, Department of Health Sciences Research, Mayo Clinic, Rochester, MN, USA

${ }^{12}$ Yale University School of Medicine, New Haven, CT, USA

${ }^{13}$ Health Research \& Educational Trust, Chicago IL, USA

${ }^{14}$ Department of Emergency Medicine, Aventura Hospital and Medical Center, Aventura, FL, USA

${ }^{15}$ Division of Endocrinology, Diabetes, Metabolism, and Nutrition, Department of Internal Medicine, Mayo Clinic, Rochester, MN, USA

We thank Erica M Ward, who assisted with formatting the manuscript and submitting it for publication, the patients who participated in our trial, and all other contributors.

Data safety monitoring board: JH (chairman; Yale University School of Medicine, New Haven, CT, USA), Chadwick Miller (Department of Emergency Medicine, Wake Forest Baptist Medical Center, Winston Salem, NC, USA), Simon Mahler (Department of Emergency Medicine, Wake Forest Baptist Medical Center), and Megan E Branda

(Department of Health Sciences Research, Division of Biostatistics and Informatics, Mayo Clinic, Rochester, MN, USA)

Contributors: The steering committee (EPH, JEH, JAK, DBD, MD, AL, NDS, JH, VMM) designed the trial, and EPH obtained funding. The site investigators (EPH, JEH, JTS, CAT, DBD, RJ, KPO, ZFM) trained and supervised research coordinators in recruiting patients and gathering the data. JH and $\mathrm{JI}$ analyzed the data. EPH and JH wrote the report. All authors had full access to all of the data (including statistical reports and tables) in the study, can take responsibility for the integrity of the data and the accuracy of the data analysis, contributed to implementation of the study and data interpretation, and approved the final report for publication. EPH is the guarantor.

Funding: Research reported in this publication was funded through a Patient-Centered Outcomes Research Institute (PCORI) award (contract 952). The views presented in this publication are solely the responsibility of the authors and do not necessarily represent the views of PCORI, its board of governors, or the methodology committee. The funder of the study had no role in the study design, data collection, data analysis, data interpretation, or writing of the report. All involved researchers' maintained independence from the funder of the study.

Competing interests: All authors have completed the ICMJE uniform disclosure form at www.icmje.org/coi disclosure.pdf and declare: JEH has research funding from Alere, Trinity, Siemens, and Roche and has consulted for Janssen. DBD has research funding from Siemens and Roche and has consulted for Janssen. All other authors have no support from any organization for the submitted work; no financial relationships with any organizations that might have an interest in the submitted work in the previous three years; no other relationships or activities that could appear to have influenced the submitted work.

Ethical approval: All study procedures were approved by the institutional review boards at each of the participating hospitals.

Data sharing: A link to the probability web tool and the "chest pain choice" decision aid can be accessed at the Mayo Clinic Shared Decision Making National Resource Center at http://shareddecisions. mayoclinic.org/decision-aid-information/chest-pain-choice-decisionaid/. Deidentified patient level data and statistical code can be requested from the corresponding author at hess.erik@mayo.edu and provided to investigators who agree to adhere to a signed research data use agreement with the Mayo Clinic.

Transparency: The corresponding author (EPH) affirms that the manuscript is honest, accurate, and a transparent account of the study being reported; that no important aspects of the study have been 
omitted; and that any discrepancies from the study as planned and registered have been explained.

This is an Open Access article distributed in accordance with the Creative Commons Attribution Non Commercial (CC BY-NC 3.0) license, which permits others to distribute, remix, adapt, build upon this work non-commercially, and license their derivative works on different terms, provided the original work is properly cited and the use is non-commercial. See: http://creativecommons.org/licenses/ by-nc/3.0/.

1 Niska R, Bhuiya F, Xu J. National Hospital Ambulatory Medical Care Survey: 2007 emergency department summary[published Online First: 2010/08/24]. Natl Health Stat Report 2010;(26):1-31.

2 Goodacre S, Cross E, Arnold J, Angelini K, Capewell S, Nicholl J. The health care burden of acute chest pain[published Online First: 2005/01/20]. Heart 2005;91:229-30. doi:10.1136/hrt.2003.027599.

3 Bhuiya FA, Pitts SR, McCaig LF. Emergency department visits for chest pain and abdominal pain: United States, 1999-2008[published Online First: 2010/09/22]. NCHS Data Brief 2010;(43):1-8.

4 Graff LG, Chern CH, Radford M. Emergency physicians' acute coronary syndrome testing threshold and diagnostic performance: acute coronary syndrome critical pathway with return visit feedback[published Online First: 2014/07/26]. Crit Pathw Cardiol 2014;13:99-103. doi:10.1097/HPC.0000000000000021.

5 Than M, Herbert M, Flaws D, et al. What is an acceptable risk of major adverse cardiac event in chest pain patients soon after discharge from the Emergency Department?: a clinical survey[published Online First: 2012/10/23]. Int J Cardiol 2013;166:752-4. doi:10.1016/j. ijcard.2012.09.171.

6 Nasrallah N, Steiner H, Hasin Y. The challenge of chest pain in the emergency room: now and the future. Eur Heart J 2011;32:656.

7 Ladapo JA, Blecker S, Douglas PS. Physician decision making and trends in the use of cardiac stress testing in the United States: an analysis of repeated cross-sectional data[published Online First: 2014/10/07] Ann Intern Med 2014;161:482-90. doi:10.7326/M14-0296.

8 Kline JA, Johnson CL, Pollack CV Ir, et al. Pretest probability assessment derived from attribute matching. BMC Med Inform Decis Mak 2005;5:26. doi:10.1186/1472-6947-5-26

9 Mitchell AM, Garvey JL, Chandra A, Diercks D, Pollack CV, Kline JA. Prospective multicenter study of quantitative pretest probability assessment to exclude acute coronary syndrome for patients evaluated in emergency department chest pain units. Ann Emerg Med 2006;47:447. doi:10.1016/j.annemergmed.2005.10.013.

10 Pierce MA, Hess EP, Kline JA, et al. The Chest Pain Choice trial: a pilot randomized trial of a decision aid for patients with chest pain in the emergency department[published Online First: 2010/05/19]. Trials 2010:11:57 doi:10.1186/1745-6215-11-57.

11 Hess EP, Knoedler MA, Shah ND, et al. The chest pain choice decision aid a randomized trial[published Online First: 2012/04/13]. Circ Cardiovasc Qual Outcomes 2012:5·251-9 doi:10.1161/CIRCOUTCOMES.111.964791.

12 Tunis SR, Stryer DB, Clancy CM. Practical clinical trials: increasing the value of clinical research for decision making in clinical and health policy. JAMA 2003:290:1624-32 doi:10.1001/jama.290.12.1624.

13 Anderson RT, Montori VM, Shah ND, et al. Effectiveness of the Chest Pain Choice decision aid in emergency department patients with low-risk chest pain: study protocol for a multicenter randomized trial[published Online First: 2014/06/03]. Trials 2014:15:166. doi:10.1186/1745-6215-15-166.

14 Kline JA, Jones AE, Shapiro NI, et al. Multicenter, randomized trial of quantitative pretest probability to reduce unnecessary medical radiation exposure in emergency department patients with chest pain and dyspnea[published Online First: 2013/11/28]. Circ Cardiovasc Imaging 2014;7:66-73. doi:10.1161/CIRCIMAGING.113.001080.

15 Karanicolas PJ, Montori VM, Devereaux PJ, Schünemann H, Guyatt GH. A new 'mechanistic-practical" framework for designing and interpreting randomized trials[published Online First: 2008/05/13]. Clin Epidemiol 2009;62:479-84. doi:10.1016/j.jclinepi.2008.02.009.

16 Fergusson D, Aaron SD, Guyatt G, Hébert P. Post-randomisation exclusions: the intention to treat principle and excluding patients from analysis[published Online First: 2002/09/21]. BMJ 2002;325:652-4. doi:10.1136/bmj.325.7365.652.

17 Pocock SI, Simon R. Sequential treatment assignment with balancing for prognostic factors in the controlled clinical trial[published Online First: 1975/03/01]. Biometrics 1975;31:103-15. doi:10.2307/2529712.

18 Hargraves I, LeBlanc A, Shah ND, Montori VM. Shared Decision Making. The Need For Patient-Clinician Conversation, Not Just Information. Health Aff (Millwood) 2016;35:627-9. doi:10.1377/hlthaff.2015.1354.

19 Montori VM, Breslin M, Maleska M, Weymiller AJ. Creating a conversation: insights from the development of a decision aid[published Online First: 2007/08/09]. PLoS Med 2007;4:e233. doi:10.1371/journal.pmed.0040233.

20 Schulz KF, Altman DG, Moher D. CONSORT Group. CONSORT 2010 statement: updated guidelines for reporting parallel group randomized trials[published Online First: 2010/03/26]. Ann Intern Med 2010;152:72632. doi:10.7326/0003-4819-152-11-201006010-00232.
21 Elwyn G, Hutchings H, Edwards A, et al. The OPTION scale: measuring the extent that clinicians involve patients in decision-making tasks. Health Expect 2005:8:34-42. doi:10.1111/j.1369-7625.2004.00311.

22 Accurint for Healthcare, LexisNexis Risk Solutions. GA, USA

23 Patient Centered Outcomes Research Institute. www.pcori.org/ about-us laccessed September 132016.

24 Weymiller AJ, Montori VM, Jones LA, et al. Helping patients with type 2 diabetes mellitus make treatment decisions: statin choice randomized trial[published Online First: 2007/05/30]. Arch Intern Med 2007:167:1076-82 doi:101001/archinte.16710.1076.

25 O'Connor AM. Validation of a decisional conflict scale[published Online First: 1995/01/01]. Med Decis Making 1995;15:25-30. doi:10.1 177/0272989X9501500105.

26 Thom DH, Ribisl KM, Stewart AL, Luke DA. The Stanford Trust Study Physicians. Further validation and reliability testing of the Trust in Physician Scale. Med Care 1999;37:510-7. doi:101097/00005650-199905000-00010.

27 Gattellari M, Ward JE. Will men attribute fault to their GP for adverse effects arising from controversial screening tests? An Australian study using scenarios about PSA screening. I Med Screen 2004;11:165-9. doi:10.1258/0969141042467386.

28 Elwyn G, Edwards A, Wensing M, Hood K, Atwell C, Grol R. Shared decision making: developing the OPTION scale for measuring patient involvement[published Online First: 2003/04/08] . Qual Saf Health Care 2003;12:93-9. doi:10.1136/qhc.12.2.93.

29 Cullen L, Than M, Brown AF, et al. Comprehensive standardized data definitions for acute coronary syndrome research in emergency departments in Australasia[published Online First: 2010/02/09]. Emerg Med Australas 2010;22:35-55. doi:10.1111/j.1742-6723.2010.01256.x.

30 Thygesen K, Alpert JS, Jaffe AS, et al. Joint ESC/ACCF/AHA/WHF Task Force for the Universal Definition of Myocardial Infarction. Third universal definition of myocardial infarction[published Online First: 2012/08/28]. Circulation 2012;126:2020-35. doi:10.1161/ CIR.0b013e31826e1058.

31 Kline JA, Zeitouni RA, Hernandez-Nino J, et alRandomized trial of computerized quantitative pretest probability in low-risk chest pain patients: effect on safety and resource use. Ann Emerg Med 2009:53.727-35 doi:S0196-0644(08)01861-1.

32 Hollander JE, Blomkalns AL, Brogan GX, et al. Multidisciplinary Standardized Reporting Criteria Task Force Standardized Reporting Criteria Working Group of Emergency Medicine Cardiac Research and Education Group-International. Standardized reporting guidelines for studies evaluating risk stratification of emergency department patients with potential acute coronary syndromes[published Online First: 2004/12/02]. Ann Emerg Med 2004;44:589-98. doi:10.1016/j. annemergmed.2004.08.009.

33 Patient Protection and Affordable Care Act, Pub L No. 111-148, 124 Stat 727, §6301.

34 Selby JV, Beal AC, Frank L. The Patient-Centered Outcomes Research Institute (PCORI) national priorities for research and initial research agenda. JAMA 2012;307:1583-4. doi:10.1001/jama.2012.500.

35 Hess EP, Grudzen CR, Thomson R, Raja AS, Carpenter CR. Shared Decision-making in the Emergency Department: Respecting Patient Autonomy When Seconds Count. Acad Emerg Med 2015; 22:856-64. doi:10.1111/acem.12703.

36 Grudzen CR, Anderson JR, Carpenter CR, Hess EP. Shared Decision Making in the Emergency Department: Development of a PolicyRelevant Patient-Centered Research Agenda. Acad Emerg Med 2016; published online 11 July 2016. doi:10.1111/acem.13047.

37 Lindor RA, Kunneman M, Hanzel M, Schuur JD, Montori VM, Sadosty AT. Liability and informed consent in the context of shared decision making. Acad Emerg Med 2016; published online 8 September 2016. doi:10.1111/acem.13078.

38 Durand MA, Moulton B, Cockle E, et alCan shared decision-making reduce medical malpractice litigation? A systematic review. BMC Health Serv Res 2015;15:167. doi:10.1186/s12913-015-0823-2.

39 Huntington B, Kuhn N. Communication gaffes: a root cause of malpractice claims. Proc (Bayl Univ Med Cent) 2003;16:157-61, discussion 161.

40 Stacey D, Légaré F, Col NF, et al. Decision aids for people facing health treatment or screening decisions. Cochrane Database Syst Rev 2014:(1):CD001431 doi:101002/14651858.CD001431 pub4.

41 McNaughton C, Wallston KA, Rothman RL, Marcovitz DE, Storrow AB. Short, subjective measures of numeracy and general health literacy in an adult emergency department. Acad Emerg Med 2011; 18:1148-55. doi:10.11/j.553-2712.011.01210.

Supplementary figure: decision aid including option of coronary computed tomography angiography

Supplementary table: knowledge questions included in post-visit patient questionnaire 